\title{
AMCoR
}

Asahikawa Medical College Repository http://amcor.asahikawa-med.ac.jp/

Parasitology International (2007) 56(4):313-316.

Molecular Characterization of a novel gene encoding an $8-k$ Da subunit of Antigen B from Echinococcus granulosus genotypes 1 and 6

Wulamu, Mamuti ; Yasuhito, Sako ; Jean-Mathieu Bart ; Minoru, Nakao ; Xiumin Ma ; Hao, Wen ; Akira, Ito 
Parasitology International 56 (2007) 313-316

\section{Molecular Characterization of a novel gene encoding an 8-kDa subunit of Antigen B from Echinococcus granulosus genotypes 1 and $6^{\S}$}

Wulamu Mamuti, ${ }^{\mathrm{a}, \mathrm{b}, \#}$, Yasuhito Sako ${ }^{\mathrm{a}, \#}$, Jean-Mathieu Bart ${ }^{\mathrm{a}, \mathrm{c}}$, Minoru Nakao ${ }^{\mathrm{a}}$, Xiumin Ma, Hao Wen ${ }^{\mathrm{d}}$, Akira Ito $^{\mathrm{a}^{*}, \#}$

${ }^{a}$ Department of Parasitology, Asahikawa Medical College, Midorigaoka Higashi 2-1-1-1, Asahikawa 078-8510, Japan

${ }^{\mathrm{b}}$ Department of Parasitology and Microbiology, Xinjiang Medical University, No. 8, Xinyi Road, Urumqi, 830054, China

' Santé Environment Rural Franche-Comté and WHO Centre Collaborating for Prevention and Treatment of Human Echinococcosis, University of Franche- Comté, 25000 Besançon, France

${ }^{\mathrm{d}}$ Xinjiang Hydatid Clinical Research Institute, No. 8, Xinyi Road Urumqi 830054, China

\footnotetext{
\# These authors worked equally.
}

${ }^{*}$ Corresponding author. Department of Parasitology, Asahikawa Medical College, Midorigaoka Higashi 2-1-1-1, Asahikawa, 078-8510, Japan. 
Tel: +81-166-68-2420 ; fax: +81-166-68-2429.

E-mail address: akiraito@asahikawa-med.ac.jp (A. Ito)

$\S$ The nucleotide and amino acid sequences of EgG1AgB8/5 and EgG6AgB8/5 have been deposited in the Genbank database with Accession Nos. $\underline{\text { AB260973 }}$ and $\underline{\text { AB260974 }}$, respectively.

\section{Abstract}

Antigen B in hydatid cyst fluid of Echinococcus granulosus is a polymeric lipoprotein of $160 \mathrm{kDa}$, and is an aggregate of several different but homologous small proteins with approximately $8 \mathrm{kDa}$ which are encoded by a multigene family. Four genes encoding 8-kDa-subunit monomers of the antigen B have been identified from E. granulosus. Recently, we have isolated another novel gene from Echinococcus multilocularis encoding a fifth 8-kDa-subunit of AgB (named EmAgB8/5), predominantly transcribed in the adult worm, but not in vesicles of metacestodes. In this study, we cloned and characterized two EmAgB8/5 homologue genes from E. granulosus genotypes 1 and 6 by PCR, and named as EgG1AgB8/5 and $E g G 6 A g B 8 / 5$, respectively. The phylogenetic relationship of these genes with other genes encoding the antigen B 8-kDa-subunit monomers was also discussed.

Keywords: Echinococcus granulosus, genotypes 1 and 6, Antigen B, 8-kDa subunit, 
EgG1AgB8/5, EgG6AgB8/5, molecular characterization.

Antigen B (AgB), initially identified in hydatid cyst fluid of Echinococcus granulosus causing cystic echinococcosis, is a major excretory-secretory antigenic component of the metacestode stage of the parasite [1], and may play an important role in host-parasite interaction during the echinococcal infections, since it is the major component of hydatid cyst fluid [2]. The E. granulosus AgB (EgAgB) is a thermostable polymeric lipoprotein of 160 kDa [1]. On SDS-PAGE, it disassociates to show a characteristic ladder-like pattern, consisting of regularly spaced subunits with apparent molecular sizes of 8, 16, 24 and $32 \mathrm{kDa}$, and the higher molecular mass subunits are supposed to be derived from the 8-kDa-subunit component [3-5]. Now it is known that the 8-kDa subunit component is encoded by a gene family (6), and at least four members (EgAgB8/1, EgAgB8/2, $E g A g B 8 / 3$ and $E g A g B 8 / 4)$ of this gene family have been identified from E. granulosus [7-10] and high degrees of sequence polymorphism of these genes have been demonstrated $[10,11]$. The sequence variability of $E g A g B$ genes is considered as the $E g A g B$ genes belong to a family of contingency genes, which might benefit an immuno-evasion mechanism of parasite in host-parasite interaction [11,12]. Recently, we have isolated five members of this family (EmAgB8/1, EmAgB8/2, EmAgB8/3, EmAgB8/4 and EmAgB8/5) from Echinococcus multilocularis causing alveolar echinococcosis, and demonstrated the developmental regulation of these genes during the $E$. multilocularis life cycle [13]. Four (EmAgB8/1, EmAgB8/2, EmAgB8/3 and EmAgB8/4) of 
them showed a high similarity to each gene isolated from E. granulosus, but EmAgB/5 had less homology to known AgB genes.

RT-PCR analyses revealed that $E m A g B 8 / 5$ is transcribed predominantly in adult worms, but not in vesicles of the E. multilocularis metacestode [13]. However, the existence of EmAgB8/5 homologue gene in E. granulosus, and its expression patterns in parasite developmental stages has been unclear. In order to better understand the genomic organization of EgAgB genes, herein we isolated two EmAgB8/5 homologue genes from E. granulosus by PCR. PCR was carried out using genomic DNA isolated from a single worm of E. granulosus genotype 1 (G1) and 6 (G6), collected from small intestines of two naturally infected dogs in Xinjiang, China [14]. A forward primer (5'-CTGGCTCTCGTAGCCTTCGTG-3') and a reverse primer (5'-GCATAAATGAATCATAATTTCTACTCC -3') derived from nucleotide sequence of EmAgB8/5 (GenBank accession No. $\underline{\text { AB202118) }}$ were applied. The reverse primer was designed from protein coding region (underlined) and 3' untranslated region (UTR). Since the 3' UTRs in each known AgB genes were highly conserved between $E$. multilocularis and E. granulosus, it has been assumed that the 3' UTR in AgB5 gene is also conserved. PCR was carried out using high fidelity polymerase, PrimeSTAR (TaKaRa, Kyoto, Japan) in a final volume of $25 \mu \mathrm{l}$ reaction mixture, containing $0.2 \mu \mathrm{M}$ of each primer, $200 \mu \mathrm{M}$ of dNTPs and 0.625 units of PrimeSTAR DNA polymerase. Amplification was performed with 35 cycles of $98^{\circ} \mathrm{C}$ for $10 \mathrm{~s}, 55^{\circ} \mathrm{C}$ for $15 \mathrm{~s}$, and $72^{\circ} \mathrm{C}$ for $20 \mathrm{~s}$, followed by a final extension at $72^{\circ} \mathrm{C}$ for $2 \mathrm{~min}$. The amplicons were excised from agarose gels using NucleoSpin 
ExTract kit (MACHEREY-NAGEL, Düren, Germany) according to the instruction manual, and were cloned into pT7Blue T-Vector (Novagen, Madison, WI, USA) after the addition of adenine to the ends of PCR products. Nucleotide sequencing was carried out using vector derived primer (T7 promoter primer \#69348-1) on an ABI PRSIM 377 Sequencer (Applied Biosystems, Foster City, CA., USA) with DYEnamic ET Terminator Cycle Sequencing Kit (Amersham Biosciences Piscataway, NJ, USA). Multiple alignments were generated by Clustal W program using BioEdit software [15]. The phylogenic tree was constructed using nucleotide sequences corresponding to mature peptides of genes isolated in this study and other AgB genes obtained from GenBank by Neighbor Joining method, and tested by bootstrap with 1000 replicates using MEGA version 3.1 [16].

Primers specific for the AgB8/5 gene generated DNA fragments of 324 bp from both $E$. granulosus genotypes. Nucleotide sequence comparison revealed that the AgB8/5 gene from E. granulosus G1 and G6, named EgG1AgB8/5 and EgG6AgB8/5, respectively, showed high identities to $E m A g B 8 / 5$ gene (Fig. 1A). In addition, genome structures with 2 exons and 1 intron were identical among three genes. Amino acid sequences of the predicted mature forms of EgG1AgB8/5 and EgG6AgB8/5 showed 96.9\% homology with that of EmAgB8/5. Two amino acid substitutions at different positions were observed compared with the amino acid sequence of EmAgB8/5; serine at position 8 was substituted to alanine in EgG1AgB8/5 and EgG6AgB8/5, while the alanine at position 34 was substituted to serine in EgG1AgB8/5 and arginine at position 60 was substituted to lysine in EgG6AgB8/5 (Fig. 1B). As expected, a 
phylogenic analysis revealed that the EgG1AgB8/5 and EgG6AgB8/5 together with

EmAgB8/5 formed a new fifth cluster in comparison with AgB tree published previously [17].

The AgB genes were expressed in a developmentally regulated manner through different

developmental stages of E. multilocularis [10] as mentioned above. This finding suggests that the differential expression of AgB genes might be relevant to different functions of the native AgB, which may composed of different 8-kDa-subunits of AgB at different developmental stages of the parasite, and may responsible to the different biological functions of this polymeric protein throughout the developmental stages of the parasite. In this study, we concentrated on to investigate the existence of EmAgB8/5 homologue gene in E. granulosus. Further analysis on expression pattern of this gene at different developmental stages of $E$. granulosus should be carried out.

In vitro experiments have demonstrated that the EgAgB has a protease inhibitor-like activity to inhibit recruitment of neutrophils [4], and to exploit activation of helper T cells by eliciting a non-protective Th2 cell response $[12,18]$. The AgB may also be involved in parasite detoxifying mechanism [19]. However, the exact biological function of this abundant protein in hydatid cyst fluid has not yet been completely understood. The findings in this study may facilitate our better understanding of protein structure as well as biological function of AgB in the host-parasite interactions during the echinococcal infection.

\section{Acknowledgements}


This study was supported in part by grant-in-aid from the Japan Society for Promotion of Science (JSPS) (14256001 and 17256002), by the US-NIH (1R01TW01565-05) (principle investigator, P.S. Craig), JSPS-Asia/Africa Science Platform Fund, and Infection Matrix Fund from the Ministry of Education, Japan (MEXT) to A. Ito.

\section{References}

[1] Oriol R, Williams JF, Esandi MVP, Oriol C. Purification of lipoprotein antigens of Echinococcus granulosus from sheep hydatid fluid. Am J Trop Med Hyg 1971;20:569-74.

[2] Musiani P, Piantelli M, Lauriola L, Arru E, Pozzuoli R. Echinococcus granulosus: specific quantification of the two most immunoreactive antigens in hydatid fluids. J Clin Pathol 1978;31:475-8.

[3] Lightowlers MW, Liu D, Haralambous A, Rickard MD. Subunit composition and specificity of major cyst fluid antigens of Echinococcus granulosus. Mol Biochem Parasitol 1989;37:171-82.

[4] González G, Nieto A, Fernándz C, Örm A, Wernstedt C, Hellman U. Two different 8 kDa monomers are involved in the oligomeric organization of the native Echinococcus granulosus antigen B. Parasite Immunol 1996;37:171-82.

[5] Monteiro KM, Scapin SMN, Navrro MVAS, Zanchin NIT, Cardoso MB, Silveira NP, et al. Self-assembly and structural characterization of Echinococcus granulosus antigen B recombinant subunit oligomers. Biochim Biophys Acta 2007; 1774: 278-285. 
[6] Chemale G, Haag KL, Ferreira HB, Zaha A. Echinococcus granulosus antigen B is encoded by a gene family. Mol Biochem Parasitol 2001;116:233-7.

[7] Shepherd JC, Aitken A, McManus DP. A protein secreted in vivo by Echinococcus granulosus inhibits elastase activity and neutrophil chemotaxis. Mol Biochem Parasitol 1991;44:81-90.

[8] Frosch P, Hartmann M, Mühlschlegel F, Frosch M. Sequence heterogeneity of the Echinococcal antigen B. Mol Biochem Parasitol 1994;64:171-5.

[9] Fernández V, Ferreira HB, Fernández C, Zaha A, Nieto A. Molecular characterization of a novel 8 kDa subunit of Echinococcus granulosus antigen B. Mol Biochem Parasitol 1996;77:247-50.

[10] Arend AC, Zaha A, Ayala FJ, Haag KL. The Echinococcus granulosus antigen B shows a high degree of genetic variability. Exp Parasitol 2004;108:76-80.

[11] Haag KL, Alves-Junior L, Zaha A, Ayala FJ. Contingent, non-neutral evolution in a multicellular parasite: natural selection and gene conversion in the Echinococcus granulosus antigen B gene family. Gene 2004;333:157-67.

[12] Rigano R, Buttari B, Profumo E, Ortona E, Delunardo F, Margutti P, et al., Echinococcus granulosus antigen B impairs human dendritic cell differentiation and polarizes immature dendritic cell maturation towards a Th2 cell response. Infect Immun 2007; 75: 1667-78. 
[13] Mamuti W, Sako Y, Xiao N, Nakaya K, Nakao M, Yamasaki H, et al. Echinococcus multilocularis: Developmental stage-specific expression of Antigen B 8-kDa-subunits. Exp Parasitol 2006;113:75-82.

[14] Bart JM, Abdukader M, Zhang YL, Lin RY, Wang YH, Nakao M, et al. Genotyping of human cystic echinococcosis in Xinjiang, PR China. Parasitology 2006;133:571-9.

[15] Hall TA. BioEdit: a user-friendly biological sequence alignment editor and analysis program for Windows 95/98/NT. Nucleic Acids Symposium Series 1999;41:95-8.

[16] Kumar S, Tamura K, Nei M. MEGA3: Integrated software for molecular evolutionary genetics analysis and sequence alignment. Briefings in Bioinformatics 2004 5:150-63.

[17] Haag KL, Zanotto PMA, Alves-Junior L, Gasser RB, Zaha A, Ayala FJ. Searching for antigen B genes and their adaptive sites in distinct strains and species of the helminth Echinococcus. Inf Genet Evol 2006;6:251-61.

[18] Riganò R, Profumo E, Bruschi F, Garulli G, Azzara A, Ioppolo S, et al. Modulation of human immune response by Echinococcus granulosus antigen B and its possible role in evading host defenses. Infect Immun 2001;69:288-96.

[19] Chemale G, Ferreira HB, Barrett J, Brophy PM, Zaha A. Echinococcus granulosus antigen B hydrophobic ligand binding properties. Biochim Biophys Acta 2005;1747: 189-94.

\section{Legends to figures:}

Fig. 1. Alignments of nucleotide (A) and deduced amino acid sequences (B) of EgG1AgB8/5 
and EgG6AgB8/5 with EmAgB8/5. The nucleotide sequences (A) are included partial exon I, intron (indicated by lower case) and partial exon II. Amino acid sequences (B) are corresponding to the mature form of the proteins deduced from exon II. The coding sequences of EmAgB8/5 are highlighted in bold. Primer regions used for cloning are indicated by dash. Multiple alignments were generated by Clustal W program using BioEdit software [12].

Fig. 2. The phylogenic tree inferred from nucleotide sequences corresponding to the mature peptides of EgG1AgB8/5, EgG6AgB8/5 and other AgB gene sequences obtained from GenBank (accession numbers are shown in the brackets). Phylogenic tree was constructed by Neighbor Joining method, and tested by bootstrap with 1000 replicates using MEGA version $3.1[13]$. 
EmAgB8/5
EgG1AgB8/5

EgG6AgB8/5

EmAgB8/5

EgG1AgB8/5

EgG6AgB8/5

EmAgB8/5

EgG1AgB8/5

EgG6AgB8/5

EmAgB8/5

EgG1AgB8/5

EgG6AgB8/5

\section{EmAgB8/5}

EgG1AgB8/5

EgG6AgB8/5

B

EmAgB8/5

EgG1AgB8/5

EgG6AgB8/5

CTGGCTCTCGTAGCCTTCGTGGCCATCGCTTTGGCgtaaattgcattataacctcccattgtgtgccaat 70

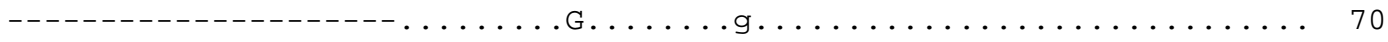

-

gcacttaaattctcacttaccettttccagGGAAGACGACATCGATTCGAAATCGAAGAAGGGTGTCA 140

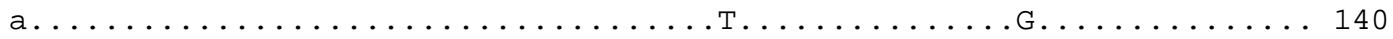

a....................................... 140

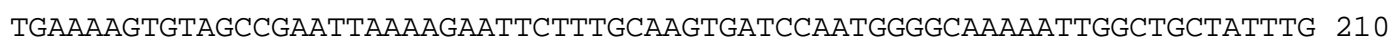
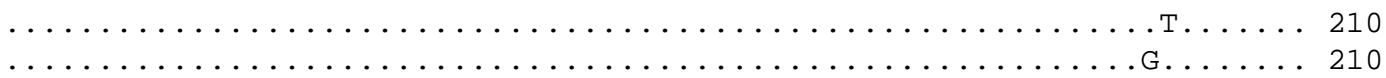

CAAGgAgCTgAAAgATTTCTTCCTTTTgGCCAgGACAAAAGCTCGCTCGgCTTTGAgAgATTATgTCAAA 280

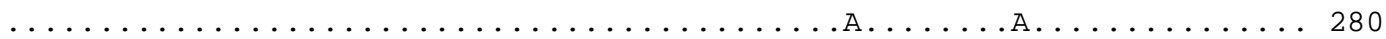

AggtTGATgGATgAAgggagtAgAAATTATgATTCATTTATGC 324

$\ldots \ldots \ldots \ldots \ldots \ldots \ldots$

.A.C............- 324

EDDIDSKSKKGVMKSVAELKEFFASDPMGQKLAAICKELKDFFLLARTKARSALRDYVKRLMDEGE 66

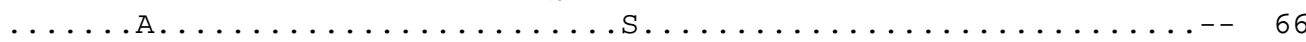

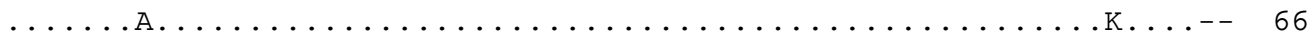

Fig. 1. Mamuti et al 


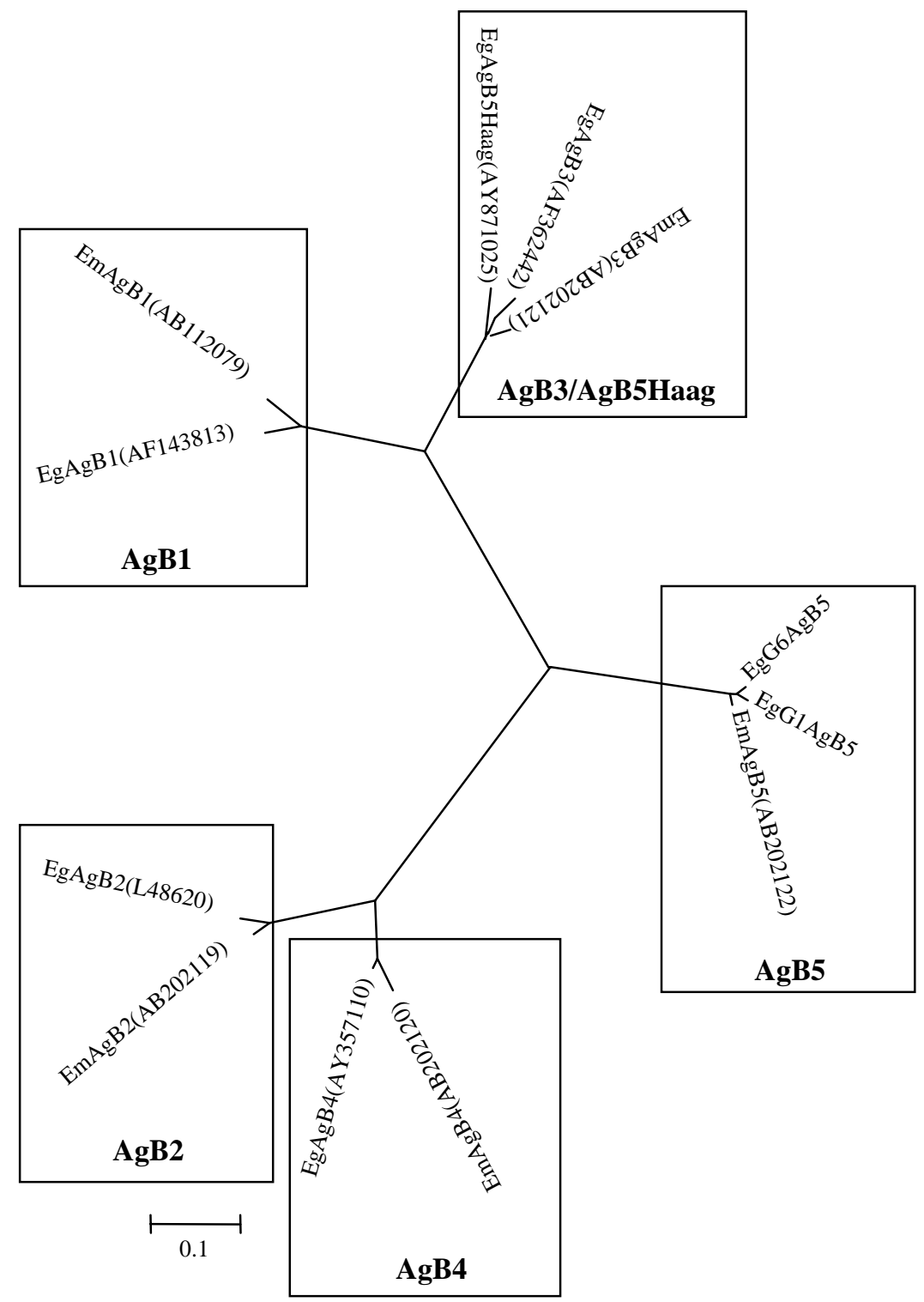

Fig. 2. Mamuti et al 